\title{
A High-Temperature Humidity Sensor Based on a Singlemode- Side Polished Multimode-Singlemode Fiber Structure
}

\author{
Xianfan Wang \\ Harbin Engineering University \\ Ke Tian \\ Harbin Engineering University \\ Libo Yuan \\ Guilin University of Electronic Technology
}

See next page for additional authors

Follow this and additional works at: https://arrow.tudublin.ie/prcart

Part of the Electrical and Computer Engineering Commons, and the Physical Sciences and Mathematics Commons

\section{Recommended Citation}

Xianfan Wang, Ke Tian, Libo Yuan, Elfed Lewis, Gerald Farrell, and Pengfei Wang, "A High-Temperature Humidity Sensor Based on a Singlemode-Side Polished Multimode-Singlemode Fiber Structure," J. Lightwave Technol. 36, 2730-2736 (2018), DOI: 10.1109/JLT.2018.2815955

This Article is brought to you for free and open access by the Photonics Research Centre at ARROW@TU Dublin. It has been accepted for inclusion in Articles by an authorized administrator of ARROW@TU Dublin. For more information, please contact arrow.admin@tudublin.ie, aisling.coyne@tudublin.ie,gerard.connolly@tudublin.ie.

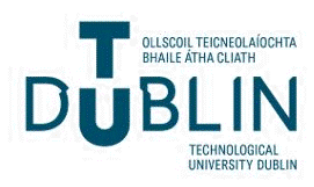




\section{Authors}

Xianfan Wang, Ke Tian, Libo Yuan, Elfed Lewis, Gerald Farrell, and Pengfei Wang

This article is available at ARROW@TU Dublin: https://arrow.tudublin.ie/prcart/28 


\title{
A High-Temperature Humidity Sensor Based on a Singlemode-Side Polished Multimode-Singlemode Fiber Structure
}

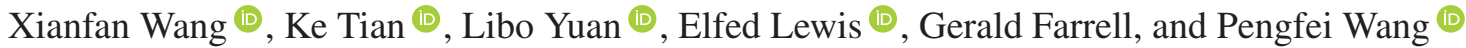

\begin{abstract}
A relative humidity (RH) sensor based on a simple singlemode-side polished multimode-singlemode (SSPMS) fiber hybrid structure is investigated, which is capable of working over a relatively high-temperature range, at which many $\mathrm{RH}$ sensors based on moisture sensitive material coatings cannot operate. The beam propagation method is used to analyze the light transmission characteristics within the side polished multimode fiber (SPMMF) structure. Experimental results show that the SPMMF surface roughness has a significant influence on the sensor's humidity sensing performance, as a result of the adsorption and desorption of water molecules along the side polished surface. A higher surface roughness results in an increased RH sensitivity. It is concluded that the SSPMS fiber structure based RH sensor can achieve around $0.069 \mathrm{~dB} / \% \mathrm{RH}$ within the humidity range of $30 \% \mathrm{RH}-90 \% \mathrm{RH}$ for a temperature range of $70{ }^{\circ} \mathrm{C}$ to 90 ${ }^{\circ} \mathrm{C}$. In addition, the temperature cross-sensitivity has been investigated experimentally. The developed fiber optic sensor in this investigation provides a simple and effective approach for $\mathrm{RH}$ measurement in a variety of production applications.
\end{abstract}

Manuscript received November 23, 2017; revised February 5, 2018 and March 6, 2018; accepted March 10, 2018. Date of publication March 15, 2018; date of current version May 15, 2018. This work was supported in part by the National Key R\&D Program of China under Grant 2016YFE0126500, in part by the National Natural Science Foundation of China under Grant 61575050, in part by the Key Program for Natural Science Foundation of Heilongjiang Province of China under Grant ZD2016012, in part by the Open Fund of the State Key Laboratory on Integrated Optoelectronics under Grant IOSKL2016KF03, in part by 111 Project (Ministry of Education China, under Grant B13015 at the Harbin Engineering University, in part by the Fundamental Research Funds of the Central University (Ministry of Education China), and in part by the Harbin Engineering University. (Corresponding author: Pengfei Wang.)

$\mathrm{X}$. Wang and K. Tian are with the Key Laboratory of In-Fiber Integrated Optics of Ministry of Education, College of Science, Harbin Engineering University, Harbin 150001, China (e-mail: heuwangfan@hrbeu.edu.cn; ketian@ hrbeu.edu.cn)

L. Yuan is with the Photonics Research Center, Guilin University of Electronic Technology, Guilin 541004, China (e-mail: lbyuan@vip.sina.com).

E. Lewis is with the Optical Fibre Sensors Research Centre, Department of Electronic and Computer Engineering, University of Limerick, Limerick V94 T9PX, Ireland (e-mail: Elfed.lewis@ul.ie).

G. Farrell is with the Key Laboratory of In-Fiber Integrated Optics of Ministry of Education, College of Science, Harbin Engineering University, Harbin 150001, China, and also with the Photonics Research Centre, Dublin Institute of Technology, Dublin 8, Ireland (e-mail: gerald.farrell@dit.ie).

P. Wang is with the Key Laboratory of In-Fiber Integrated Optics of Ministry of Education, College of Science, Harbin Engineering University, Harbin 150001, China and also with the Key Laboratory of Optoelectronic Devices and Systems of Ministry of Education and Guangdong Province, College of Optoelectronic Engineering, Shenzhen University, Shenzhen 518060, China (e-mail: pengfei.wang@dit.ie).

Color versions of one or more of the figures in this paper are available online at http://ieeexplore.iee.org.

Digital Object Identifier 10.1109/JLT.2018.2815955
Index Terms-Humidity sensor, high temperature, side polished fiber, surface roughness.

\section{INTRODUCTION}

A CCURATE RH measurement plays an important role in a wide variety of areas including weather forecasting, food processing, air conditioning adjustment and industrial production. Compared with conventional humidity sensors such as electronic humidity sensors [1], optical fiber humidity sensors can offer some unique advantages, such as compact size, immunity to electromagnetic interference, and resistance to harsh environments. In the past few decades, optical fiber humidity sensors have attracted significant research interest, for example those based on a fiber Bragg grating (FBG) [2], long period grating (LPG) [3], plastic optical fiber (POF) [4], tapered optical fiber (TOF) [5], photonic crystal fiber (PCF) [6] and side polished fiber (SPF) [7]. Taking into consideration factors such as cost, fabrication process complexity, mechanical strength and stability, SPF based sensors have the potential to be a good choice for humidity sensing.

Side polished silica singlemode fiber (SPF) structures have been successfully developed into a wide variety of sensors, including those for refractive index (RI) [8], temperature [9], RH [7] and gas concentration [10]. However, in order to achieve good sensing performance, a sensor based only on singlemode fiber (SMF) must be side polished with enough depth to expose the fiber core to the external environment, which can greatly increase the risk of breakage, which in turn limits the practical application of the SPF structures as sensors. It is worth noting that side polished plastic optical fiber (SP-POF) structures have been investigated widely due to their inherent flexibility, tensile strength and relatively easy micro-fabrication [11]. However, their intrinsic material properties hinder their application in physical parameter sensing and furthermore temperature induced variations for SP-POF structures cannot be neglected. Therefore, some additional polymer coating or other processing means have to be employed to implement physical parameter sensing and mitigate the effect of temperature, which in turn increases fabrication cost and difficulty.

To date, there has been little research published on singlemode-side polished multimode-singlemode (SSPMS) fiber structure based humidity sensors [12]. It is widely recognised that a singlemode-multimode-singlemode (SMS) fiber 
structure is a relatively simple structure, comprising a short length of multimode fiber (MMF) sandwiched between two standard singlemode fibers (SMFs) [13]. Multimode interference (MMI) phenomena within the MMF section provides a useful basis for developing a variety of fiber optic devices and sensors, e.g., bandpass filters [14], edge filters [15], RI sensors [16], temperature sensors [17], RH sensors [18] and curvature sensors [19]. However, for most of the existing sensors based on an SMS fiber structure, it is necessary to remove the MMF cladding completely to expose its core to the surrounding environment. Common fabrication methods include hydrofluoric acid etching [16], tapering the MMF to micrometer scale [20], misalignment between the input SMF and MMF [21] and side polishing the MMF [22]. Since other methods either require expensive fabrication steps or employ potentially toxic/unhealthy experimental materials, side polishing the MMF has the potential to be an appropriate candidate for fabricating SSPMS fiber structure based sensors, with the advantage that a sensor based on SPMMF in an SMS structure necessitates the removal of far less silica glass, compared to sensors based on SPF only, reducing fragility and the risk of breakage.

In addition, for many optical fiber based humidity sensors, several humidity sensitive materials must be used, mainly as coatings to improve the RH sensing performance of fiber optic sensors. For example in previously reported work a variety of polymer coatings were utilized, e.g., polyvinyl alcohol (PVA) [23], agarose [24], gelatin [25], polymethyl methacrylate (PMMA) [26], polyethylene oxide (PEO) [27] as well as other nanocoatings [28], [29]. However, these additional coatings including organic and inorganic materials, also result in some disadvantages such as a limited temperature range for operation, high price and additional fabrication complexity. The humidity sensor of this investigation is based on adsorption and desorption of moisture only on the silica fiber surface. In addition, this sensor provides the potential for operation even at superheated temperatures (above $100{ }^{\circ} \mathrm{C}$ ) due to the exclusive use of silica as the only sensor material. The influence of different surface roughness on the humidity sensitivity of the SSPMS fiber structure based on adsorption and desorption of moisture has also been investigated.

In this paper an SSPMS fiber structure with a deliberate surface roughness for the side polished area is investigated as an RH sensor. Firstly, the beam propagation method (BPM) was employed to model and analyze the influence of side polishing on light transmission within the fiber structure. The sensing mechanism for the SSPMS fiber structure is also introduced. The fabrication process of such an SSPMS fiber structure is described in detail, involving a method of producing different side polished surface roughness levels. The SPMMF surfaces of three fabricated SSPMS fiber structure samples were characterized employing a scanning electron microscope (SEM). From a comparison of the RH sensitivities and temperature dependence of these fiber samples within an $\mathrm{RH}$ range of $30 \% \mathrm{RH}-90 \% \mathrm{RH}$, it is established that the sensitivity of the SSPMS fiber structure based RH sensors is related to their side polished fiber surface roughness, and a coarser side polish surface results in a higher $\mathrm{RH}$ sensitivity in the operating temperature range $70{ }^{\circ} \mathrm{C}-90^{\circ} \mathrm{C}$.
Furthermore, a relationship between RH sensitivity and side polished surface roughness level is presented. Finally, these fiber optic RH sensors are also demonstrated to have a low temperature dependence.

\section{DESIGN AND PRINCIPLE}

It is well established that a conventional SMS fiber structure comprises a short length of MMF sandwiched between two SMFs, within which the MMF is circularly symmetrical. The light propagation characteristics within the MMF structure has been widely investigated in previous papers [30]. However, the MMF employed in this investigation within the SSPMS fiber structure does not possess the characteristic of circular symmetry, because its entire cladding and part of the core are removed by polishing on one side. According to our previous work [12], the SPMMF comprises two lead-in/lead-out transitional regions and a side polished flat region. The fabricated SPMMF section can be seen as multimode waveguide surrounded with air. When the light is launched from the lead-in SMF to the MMF, many higher modes are excited. Here, the input light is assumed to have a field distribution of $E(x, y, 0)$, and the excited eigenmodes within the SPMMF can be denoted as $F_{m}(x, y)$. If the radiation loss within the SPMMF is not considered, we have

$$
E(x, y, 0)=\sum_{m=1}^{M} a_{m} F_{m}(x, y)
$$

where $M$ is total number of the excited modes, and $a_{m}$ is excitation coefficient, and it can be calculated as

$$
a_{m}=\frac{\int_{0}^{\infty} \int_{0}^{\infty} E(x, y, 0) F_{m}(x, y) d x d y}{\int_{0}^{\infty} \int_{0}^{\infty} F_{m}(x, y) F_{m}(x, y) d x d y}
$$

Thus, we can obtain the field distribution at the propagation distance $z$, which is expressed as

$$
E(x, y, z)=\sum_{m=1}^{M} a_{m} F_{m}(x, y) \exp \left(i \beta_{m} z\right)
$$

where $\beta_{m}$ is propagation constant. It is significant that the field distribution evolves along the axial propagation distance as a result of MMI occurred within the SPMMF. When the light reaches the lead-out SMF, the eigenmodes of the SPMMF are coupled into the fundamental mode of the output SMF. This SPMMF model and the beam propagation method are employed to simulate the light transmission characteristics within the SPMMF. Fig. 1 illustrates the simulated amplitude distribution of the propagating light within an SMS fiber structure and an SPMMF structure. From a comparison between Fig. 1(a) and Fig. 1(b), it can be determined that the side polishing process has a great influence on the light transmission within the SPMMF. The interaction between the evanescent field and the surrounding environment occurs due to the absence of the part of the cladding and coupling of the light between this and the core.

However, the SSPMS fiber structure is fabricated using a standard fiber optic side polishing system, which means that the side polished fiber surface of the fabricated SSPMS fiber structure is not completely smooth. As a result there are many micropores 


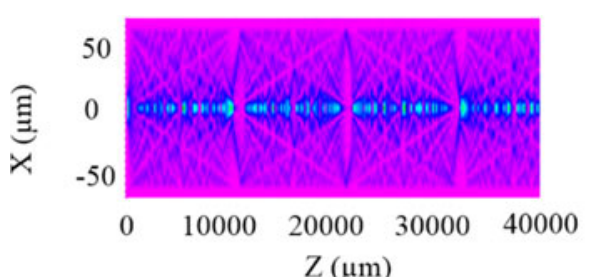

(a)

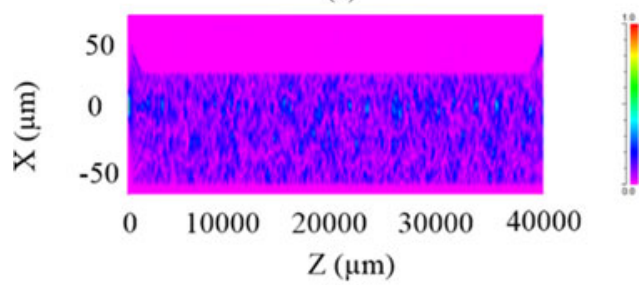

(b)

Fig. 1. The optical amplitude (intensity) distribution along the propagation direction of (a) an MMF structure; (b) an SPMMF structure.

on the fiber surface, where the morphology of the micropores is related to the grade of the abrasive paper used. When the RH of the surrounding environment increases, water molecules are adsorbed in the micropores or on the side polished fiber surface. Based on water adsorption isotherms theory, the amount of water molecules adsorbed depends on the surrounding environmental $\mathrm{RH}$ values. The water adsorption isotherms are also related to the size and shape of the micropores. For relatively coarse side polished fiber surface, a larger number and larger micropores diameters are created, compared with the relatively smooth side polished fiber surface. Therefore, it is clear that more water molecules are adsorbed on the relatively coarse side polished fiber surface when the RH increases. In a manner similar to the humidity sensing mechanisms demonstrated in [31], the effective refractive index of the guided modes within the SPMMF is altered by adsorption and desorption of water molecules on the side polished fiber surface or in the micropores of the fiber structure. As the adsorption water molecules increases in the micropores of the fiber structure, the effective refractive index of guided mode within SPMMF increases, which results in an interference dip or peak wavelength shift. Additionally, when the RH increases, water molecules adsorbed start condensing within the micropores and on the side polished fiber surface, and as the RH increases further to a high value the SPMMF section is effectively completely surrounded by a water cladding. Compared with air cladding, the water cladding formed around the SPMMF results in a stronger evanescent field. Therefore, the transmitted power decreases with respect to the $\mathrm{RH}$, as the $\mathrm{RH}$ increases. The next section describes an experimental investigation of the sensing performance of the SSPMS fiber structure based RH sensors with different side polished fiber surface roughness values.

\section{EXPERIMENT}

In the experiments of this investigation, the SMF and MMF used are SMF 28 and FG105LCA respectively. Firstly, an SMS fiber structure was constructed by sandwiching a $4 \mathrm{~cm}$ length

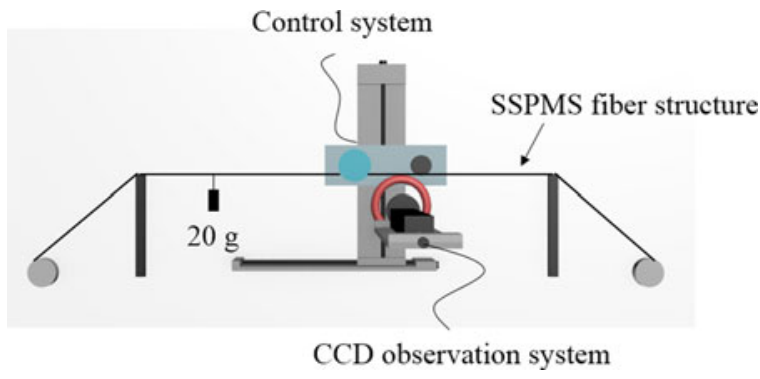

Fig. 2. Experimental setup for a fiber optic side polish system.

of MMF between two SMFs utilizing a standard precision fiber cleaver (Fujikura CT-32) and fusion splicer (Fujikura 62S). A length of $4 \mathrm{~cm}$ for the MMF section was chosen based on the lengths previously utilized for similar SMS structures [16]. A fiber optic side polishing system was then used to side polish the SMS fibe structure to form an SSPMS fiber structure, as depicted in Fig. 2. This system comprises a control system, a CCD camera observation system and an operation (actuation) portion. The aforementioned SMS fiber structure was rigidly fixed between two holders and tied to two standing pulleys to keep it straight. In addition, a 20-g weight was hung over this fiber structure to produce tension in the fibre structure in case of accidental breakage during the polishing process. An abrasive paper coated grinding wheel was attached to three displacement stages, whose position relative to the fibre was accurately controlled using a LABVIEW program to predetermine the side polished length and depth. The real-time condition of the side polished section of the SMS was actively monitored using the CCD camera.

Note that for the side polished fiber a desired surface roughness can be achieved by utilizing different grades of abrasive papers. In this experiment, three SSPMS fiber samples were fabricated using 800-mesh, 1600-mesh, and 12000-mesh abrasive papers, and were identified as SSPMS 1, SSPMS 2, and SSPMS 3 respectively. The side polished depth and length of these three SSPMS samples were respectively $40 \mu \mathrm{m}$ and $4 \mathrm{~cm}$, which is the same as in a previous paper by some of the authors of this article. Subsequently, a scanning electron microscope (SEM) was used to characterize the side polished surface of the three SSPMS samples. Fig. 3(a)-(c) shows the surface SEM images of the SSPMS 1, SSPMS 2 and SSPMS 3 fiber samples respectively. It is clear from comparison among Fig. 3(a)-(c) that the SSPMS 1 sample possesses the roughest side polished fiber surface while SSPMS 3 possesses the smoothest side surface, which corresponds to the grade of the abrasive papers used.

In this case, a reasonable level of repeatability of the fabrication process was also achieved. Three SSPMS fiber structures, of which the length and side polished depth of the side polished multimode fiber were predetermined as $4 \mathrm{~cm}$ and $40 \mu \mathrm{m}$ respectively, were fabricated using 800-mesh abrasive papers, and identified as SSPMS a, SSPMS b and SSPMS c. By connecting the SSPMS fiber structures with a supercontiuum source (YSL SC-series) and an optical spectrum analyzer (YOKOGAWA AQ6370D), the measured transmission spectrums were obtained, as shown in Fig. 4. From this, it was determined that the three transmission spectrums have a common overall shape 


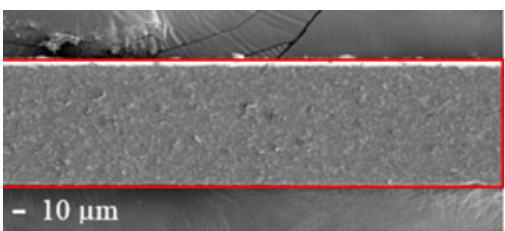

(a)

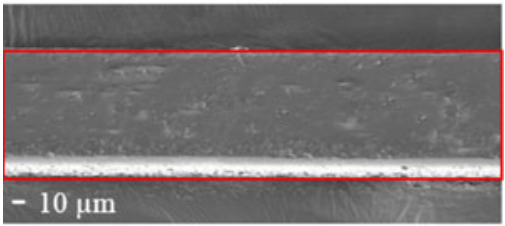

(b)

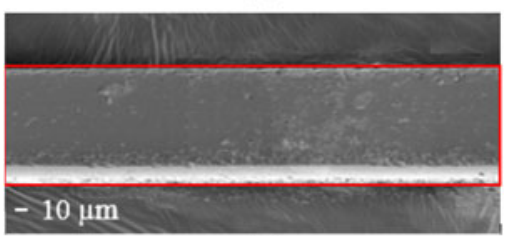

(c)

Fig. 3. SEM images of side polished surface of (a) SSPMS 1; (b) SSPMS 2; and (c) SSPMS 3 fiber sample, and the red lines illuminate the shapes of the fiber samples.

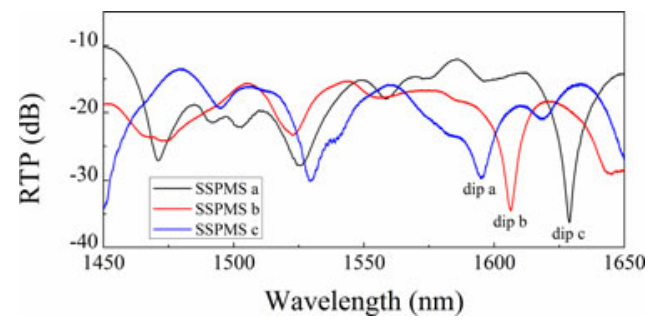

Fig. 4. Measured transmission spectrums of three SSPMS fiber structures fabricated with 800-mesh abrasive papers.

over the wavelength range of $1450 \mathrm{~nm}$ to $1650 \mathrm{~nm}$, which is recognized as an indicator of good repeatability of the fabrication process. However, it is acknowledged that a difference exists between the interference dips (dip a, dip b and dip c) which can be attributed to current limitations of reproducibility of the devices of this investigation.

To investigate the influence of surface roughness on humidity sensing at a relatively high temperature, the three fabricated SSPMS fiber samples were tested in a climate chamber (ESPEC $\mathrm{SH}-222)$, in which the $\mathrm{RH}$ and temperature were accurately controlled within a temperature range of $-20^{\circ} \mathrm{C}$ to $150^{\circ} \mathrm{C}$ and a relative humidity range of $30-95 \% \mathrm{RH}$. The climate chamber used in this investigation includes an automatic feedback control system, in which the temperature and relative humidity values can be monitored on the front display panel. Because this chamber needs several minutes to reach the input values and then stabilise, the experimental results were not recorded until the displayed values reached their correct steady state values. Fig. 5 illustrates the experimental setup for RH sensing of the fabricated SSPMS fiber samples. A supercontinuum source and an optical spectrum analyzer were used for launching the $700 \mathrm{~nm}-1700 \mathrm{~nm}$ light signal and for collecting and recording

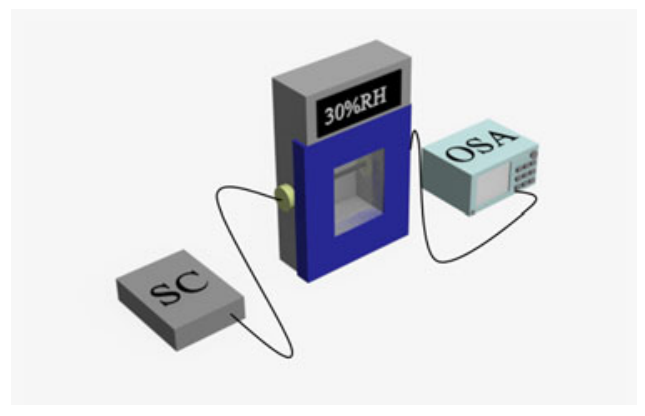

Fig. 5. Experimental setup for humidity measurement on an SSPMS fiber structure.

the output light spectrum, respectively. In order to minimize unwanted motion or vibration induced signal fluctuations, the SSPMS fiber samples were glued directly on a silicon substrate using UV curing adhesive. In addition, the climate chamber in this experiment could not be effectively used to control the $\mathrm{RH}$ value when the temperature was greater than $100{ }^{\circ} \mathrm{C}$. Therefore, RH sensitivity of three SSPMS fiber samples could only be investigated within the temperature range of $70^{\circ} \mathrm{C}-90{ }^{\circ} \mathrm{C}$.

\section{RESUlTS AND DisCUSSION}

For the three SSPMS fiber samples, the RH induced transmission spectral variations were experimentally investigated, with the temperature set to $70{ }^{\circ} \mathrm{C}, 80^{\circ} \mathrm{C}$ and $90^{\circ} \mathrm{C}$. In order to obtain more accurate experimental results, the relative transmission power (RTP) was adopted to characterize the response of our proposed humidity sensor to the ambient humidity variation, which is calculated by removing the inherent fluctuations of the supercontinuum source from the OSA measured transmission spectrum. Fig. 6(a)-(c) represent the RTP of the SSPMS 1 sample at the wavelength centered about $1595 \mathrm{~nm}$ when the RH value was changed from $30 \% \mathrm{RH}$ to $90 \% \mathrm{RH}$ with an increment of $10 \%$ RH. From these figures, it can be seen that there exists a clear trend that the RTP at the interference dip wavelength decreases with RH. Furthermore, Fig. 6(d) shows an excellent linear relationship between measured RTPs and RH values for the three temperatures with an $\mathrm{R}^{2}$ value in the range 0.974 to 0.996. When the temperatures are respectively $70{ }^{\circ} \mathrm{C}, 80{ }^{\circ} \mathrm{C}$ and $90{ }^{\circ} \mathrm{C}$, the corresponding sensitivities are measured to be $-0.069 \mathrm{~dB} / \% \mathrm{RH},-0.071 \mathrm{~dB} / \% \mathrm{RH}$ and $-0.067 \mathrm{~dB} / \% \mathrm{RH}$. The results of a linear regression performed on the data of Fig. 6(d) shows that the linear regression coefficients $\left(\mathrm{R}^{2}\right)$ are in the range 0.974 to 0.996 , which is indicative of very good linearity.

Similarly, the fabricated SSPMS 2 sample and SSPMS 3 sample were investigated under identical experimental conditions as the SSPMS 1 sample. The obtained experimental results are presented in Figs. 7 and 8. Fig. 7(a)-(c) illustrate the RTP within the $\mathrm{RH}$ range of $30 \% \mathrm{RH}-90 \% \mathrm{RH}$, when the temperatures were fixed at $70{ }^{\circ} \mathrm{C}, 80{ }^{\circ} \mathrm{C}$ and $90{ }^{\circ} \mathrm{C}$ respectively. In Fig. 7(d), it is clear that there exists a linear relationship between RTPs at the interference wavelength (circa $1550 \mathrm{~nm}$ ) and $\mathrm{RH}$ values. The calculated sensitivities are respectively $-0.038 \mathrm{~dB} / \% \mathrm{RH}$, $-0.038 \mathrm{~dB} / \% \mathrm{RH}$ and $-0.038 \mathrm{~dB} / \% \mathrm{RH}$ at the fixed temperature values of $70{ }^{\circ} \mathrm{C}, 80{ }^{\circ} \mathrm{C}$ and $90{ }^{\circ} \mathrm{C}$, and the 


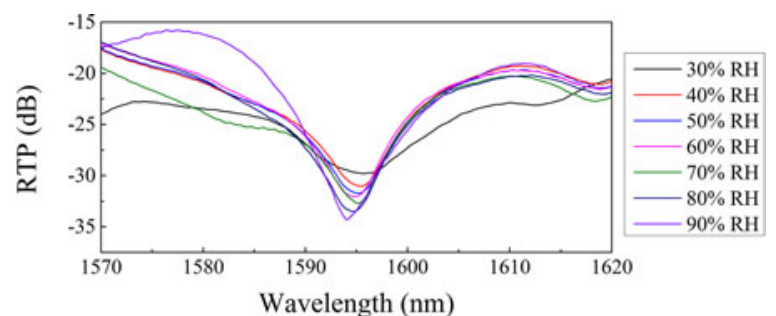

(a)

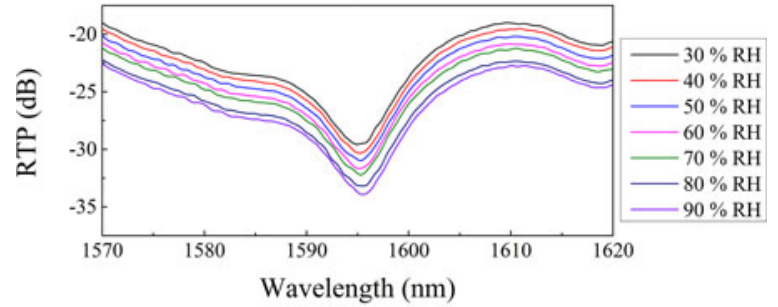

(b)

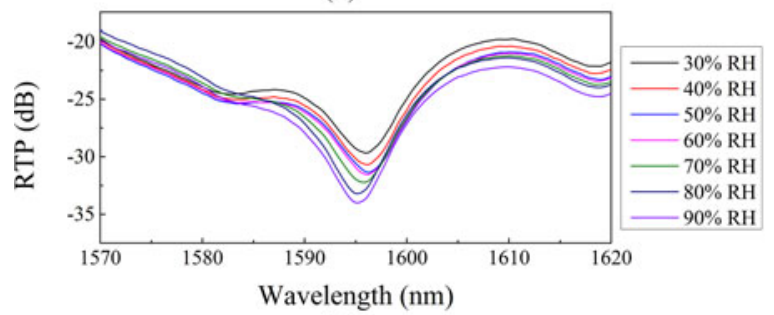

(c)

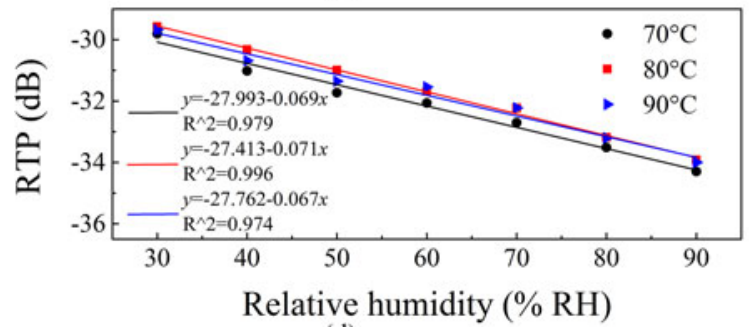

(d)

Fig. 6. Measured transmission spectral response of the SSPMS 1 within the humidity range of $30 \% \mathrm{RH}-9 \% \mathrm{RH}$ under temperature of (a) $70{ }^{\circ} \mathrm{C}$; (b) $80{ }^{\circ} \mathrm{C}$ and (c) $90^{\circ} \mathrm{C}$; (d) humidity sensitivity.

corresponding linear regression coefficients $\left(\mathrm{R}^{2}\right)$ are respectively $0.999,0.996$ and 0.996 , which is again indicative of excellent linearity. In addition, it is significant from Fig. 8(a)-(c) that the RTPs at the interference wavelength (circa $1627 \mathrm{~nm}$ ) decrease with respect to the increasing RH values. Fig. 8(d) indicates the measured $\mathrm{RH}$ sensitivities of $-0.029 \mathrm{~dB} / \% \mathrm{RH}$, $-0.028 \mathrm{~dB} / \% \mathrm{RH}$ and $-0.031 \mathrm{~dB} / \% \mathrm{RH}$ at different temperatures of $70{ }^{\circ} \mathrm{C}, 80{ }^{\circ} \mathrm{C}$ and $90{ }^{\circ} \mathrm{C}$, accompanied with a good linearity ( $\mathrm{R}^{2}$ values) of $0.997,0.998$ and 0.998 , respectively.

The RH sensing performance of the three SSPMS samples are included and compared in Table I. From this, it is clear that the measured RH sensitivities for each of the sensing samples exhibit small variations for different temperatures of $70{ }^{\circ} \mathrm{C}, 80{ }^{\circ} \mathrm{C}$ and $90{ }^{\circ} \mathrm{C}$. On the other hand, the RH sensitivities of SSPMS samples show a much stronger dependence on the surface roughness as expected. The experimental results are in good agreement with the theoretical analysis of this investigation, which illustrates that the rougher side polished fiber surface can result in more water molecules being adsorbed on

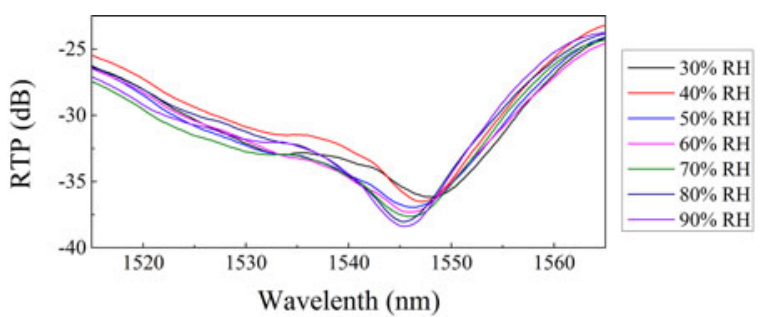

(a)
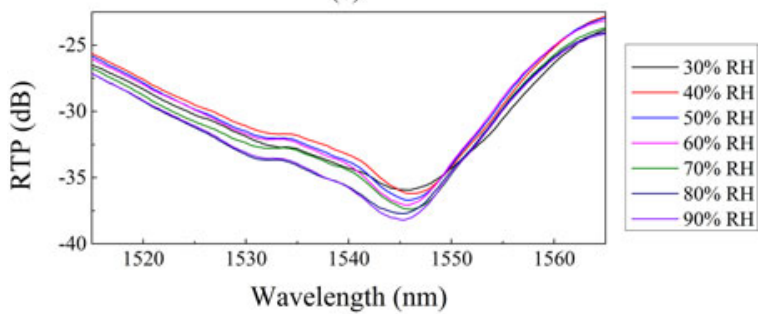

(b)

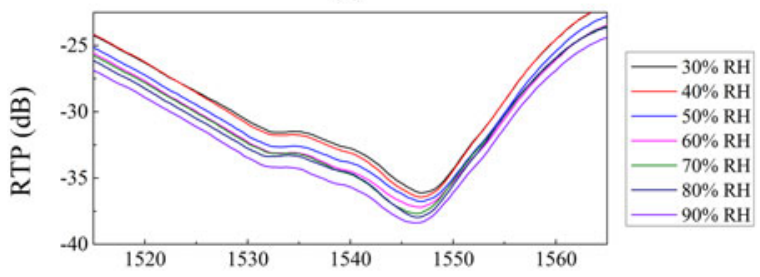

(c)

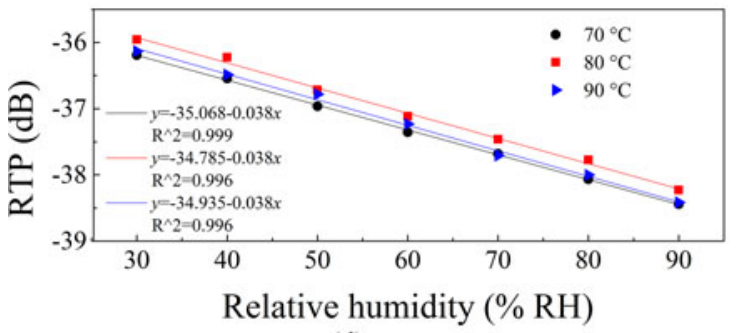

(d)

Fig. 7. Measured transmission spectral response of the SSPMS 2 within the humidity range of $30 \% \mathrm{RH}-90 \% \mathrm{RH}$ under temperature of (a) $70{ }^{\circ} \mathrm{C}$; (b) $80{ }^{\circ} \mathrm{C}$ and (c) $90{ }^{\circ} \mathrm{C}$; (d) humidity sensitivity.

TABLE I

RH SENSITIVITY OF THE INVESTIGATED SSPMS 1, SSPMS 2 AND SSPMS 3 UNDER DiFFERENT TEMPERATURES OF $70{ }^{\circ} \mathrm{C}, 80^{\circ} \mathrm{C}, 90^{\circ} \mathrm{C}$

\begin{tabular}{cccccc}
\hline \hline & & \multicolumn{4}{c}{$\mathrm{RH}$ sensitivity $(\mathrm{dB} / \% \mathrm{RH})$} \\
\hline \hline Sample & $\begin{array}{c}\text { Abrasive } \\
\text { papers }\end{array}$ & $70{ }^{\circ} \mathrm{C}$ & $80{ }^{\circ} \mathrm{C}$ & $90{ }^{\circ} \mathrm{C}$ & $\begin{array}{c}\text { Average } \\
\text { sensitivity }\end{array}$ \\
\hline SSPMS 1 & 800 -mesh & -0.069 & -0.071 & -0.067 & -0.069 \\
SSPMS 2 & 1600 -mesh & -0.038 & -0.038 & -0.038 & -0.038 \\
SSPMS 3 & 12000 -mesh & -0.029 & -0.028 & -0.031 & -0.029 \\
\hline \hline
\end{tabular}

the side polished fiber surface or within the micropores of the fiber structure. A more accurate relationship between $\mathrm{RH}$ sensing performance and side polished fiber surface roughness can be investigated further based on an analysis of the micropores size on the side polished fiber surface.

Temperature dependence is a significant factor when designing an RH sensor for practical applications. The same experimental setup was used to investigate the temperature induced optical spectrum variation. In this case, the RH value of the climate chamber was fixed at $30 \% \mathrm{RH}$, and the influence of 


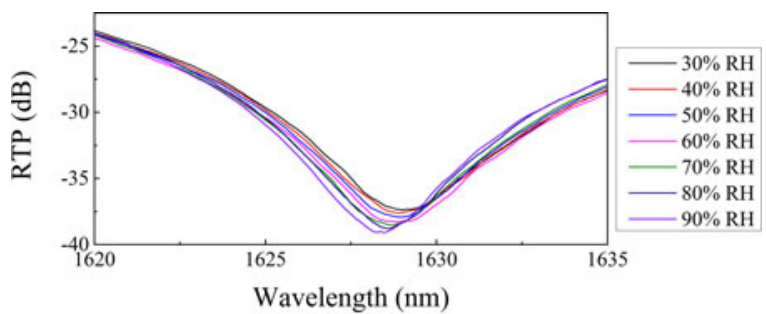

(a)

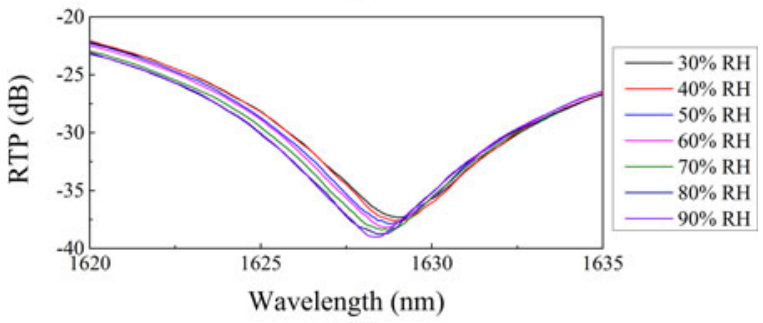

(b)

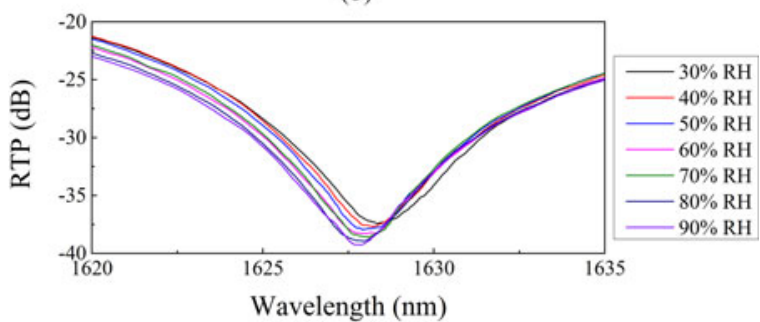

(c)

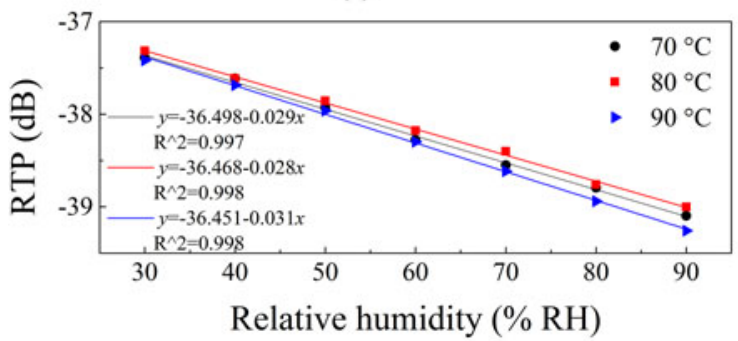

(d)

Fig. 8. Measured transmission spectral response of the SSPMS 3 within the humidity range of $30 \%$ RH- $90 \%$ RH under temperature of (a) $70{ }^{\circ} \mathrm{C}$; (b) $80^{\circ} \mathrm{C}$ and (c) $90{ }^{\circ} \mathrm{C}$; (d) humidity sensitivity.

temperature on the transmission spectra is shown in Fig. 9. Fig. 9(a)-(c) respectively present the transmission spectrum evolution within temperature range from $30{ }^{\circ} \mathrm{C}$ to $90{ }^{\circ} \mathrm{C}$ for SSPMS 1, SSPMS 2 and SSPMS 3. Furthermore, the relationship between RTP of interference dip wavelength and temperature is presented as Fig. 9(d). The black scatter dots represent the RPT values under different temperatures for SSPMS 1, while the black line represents the average RTP value for SSPMS 1. Similarly, the red scatter dots and red line represent the corresponding values for SSPMS 2, while the blue scatter dots and blue line represent the corresponding values for SSPMS 3. From this, it was determined that within temperature range of $30^{\circ} \mathrm{C}$ to $90^{\circ} \mathrm{C}$, the maximum difference between the RTP values and average value is less than $0.15 \mathrm{~dB}$, This represents an RH uncertainty in the range $2 \%$ to $10 \% \mathrm{RH}$, which can be mainly attributes to the temperature-optic effect and temperature expansion effect of the intrinsic fiber structure.

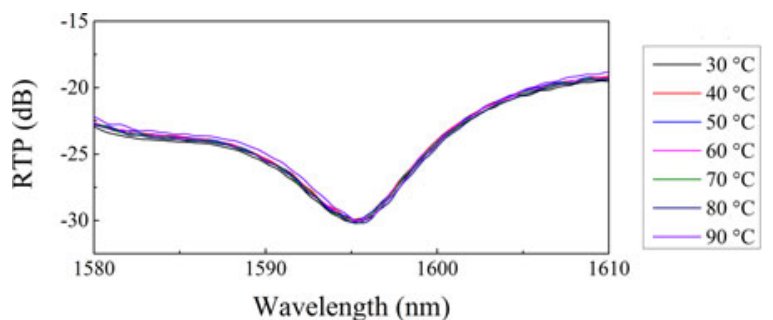

(a)

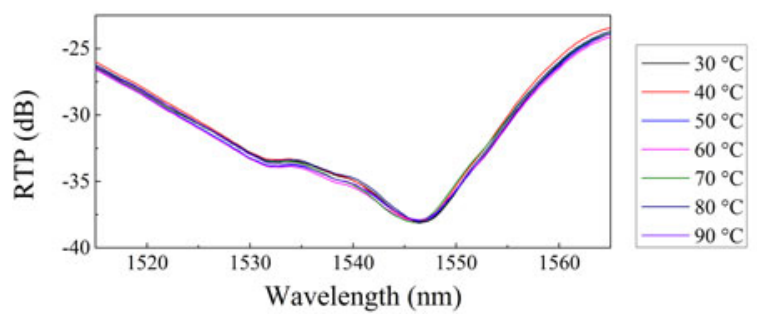

(b)

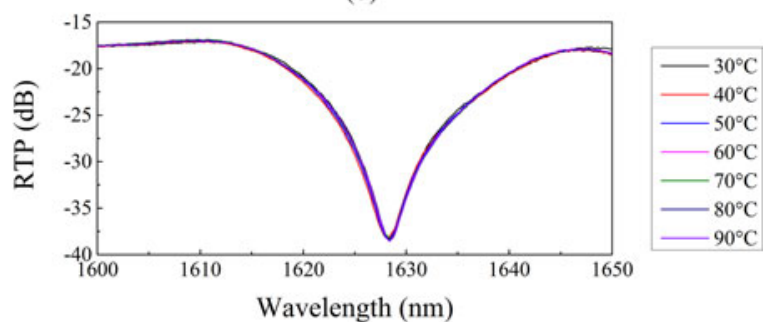

(c)

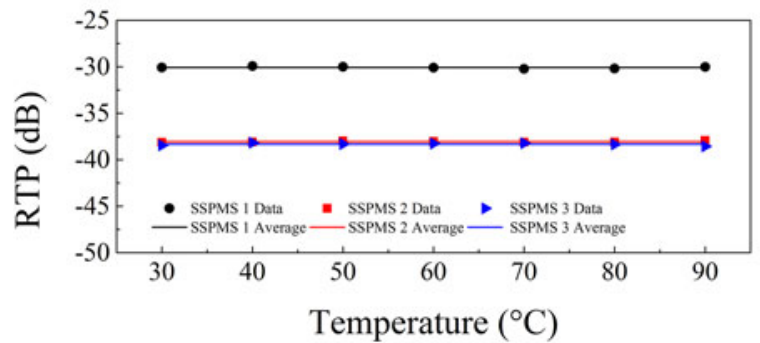

(d)

Fig. 9. Temperature response of SSPMS fiber samples. (a), (b) and (c) represent transmission spectrum with temperature increasing from $30{ }^{\circ} \mathrm{C}$ to $90{ }^{\circ} \mathrm{C}$ for SSPMS 1, SSPMS 2 and SSPMS 3 fiber sample respectively; (d) represent relationship between transmission loss and temperature for SSPMS 1, SSPMS 2 and SSPMS 3 fiber sample.

\section{CONCLUSION}

A low cost optical fiber RH sensor which does not require a polymer coating has been reported in this paper. A novel but simple fabrication method for the SSPMS fiber structure with different surface roughness was introduced, and three SSPMS sensor structures based on using 800-mesh, 1600-meh and 12000mesh abrasive papers were characterized using SEM images. Experimental results have shown that the different SSPMS samples fabricated in this investigation have RH sensitivities of $-0.069 \mathrm{~dB} / \% \mathrm{RH},-0.038 \mathrm{~dB} / \% \mathrm{RH}$ and $-0.029 \mathrm{~dB} / \% \mathrm{RH}$, which is related to the observed surface roughness. This demonstrates that deliberately abrading to achieve a rougher surface can improve the sensitivity of SSPMS fiber structure based RH sensors. Without the polymer coating limitation, the developed humidity sensors can operate well in a relatively high temperature environment $\left(70{ }^{\circ} \mathrm{C}, 80{ }^{\circ} \mathrm{C}\right.$ and $\left.90^{\circ} \mathrm{C}\right)$, under which some 
previously reported humidity sensors have exhibited rapid and catastrophic deterioration. In addition, the sensors developed in this investigation were highly linear in their response in the temperature range of $30^{\circ} \mathrm{C}-90^{\circ} \mathrm{C}$ both in terms of the shape of the output spectrum and the depth of the absorption peak. This optical fiber structure based on side polished surface roughness may provide a means to develop excellent and robust sensors for a variety of applications.

\section{REFERENCES}

[1] N. Yamazoe and Y. Shimizu, "Humidity sensors: Principles and applications," Sensors Actuators, vol. 10, no. 3/4, pp. 379-398, 1986.

[2] T. Yeo, T. Sun, K. Grattan, D. Parry, R. Lade, and B. Powell, "Characterisation of a polymer-coated fibre Bragg grating sensor for relative humidity sensing," Sensors Actuators B, Chem., vol. 110, no. 1, pp. 148-156, 2005.

[3] T. Venugopalan, T. Sun, and K. Grattan, "Long period grating-based humidity sensor for potential structural health monitoring," Sensors Actuators A, Phys., vol. 148, no. 1, pp. 57-62, 2008.

[4] S. Muto, O. Suzuki, T. Amano, and M. Morisawa, "A plastic optical fibre sensor for real-time humidity monitoring," Meas. Sci. Technol., vol. 14, no. 6, pp. 746-750, 2003.

[5] C. Bariain, I. R. Matıas, F. J. Arregui, and M. López-Amo, "Optical fiber humidity sensor based on a tapered fiber coated with agarose gel," Sensors Actuators B, Chem., vol. 69, no. 1, pp. 127-131, 2000.

[6] T. Li, X. Dong, C. C. Chan, K. Ni, S. Zhang, and P. P. Shum, "Humidity sensor with a PVA-coated photonic crystal fiber interferometer," IEEE Sensors J., vol. 13, no. 6, pp. 2214-2216, Jun. 2013.

[7] A. Alvarez-Herrero, H. Guerrero, and D. Levy, "High-sensitivity sensor of low relative humidity based on overlay on side-polished fibers," IEEE Sensors J., vol. 4, no. 1, pp. 52-56, Feb. 2004.

[8] L. Bilro, N. J. Alberto, L. M. Sa, J. de Lemos Pinto, and R. Nogueira, "Analytical analysis of side-polished plastic optical fiber as curvature and refractive index sensor," J. Lightw. Technol., vol. 29, no. 6, pp. 864-870, Mar. 2011

[9] J. Senosiain, I. Díaz, A. Gastón, and J. Sevilla, "High sensitivity temperature sensor based on side-polished optical fiber," IEEE Trans. Instrum. Meas., vol. 50, no. 6, pp. 1656-1660, Dec. 2001.

[10] A. O. Dikovska, P. Atanasov, A. T. Andreev, B. Zafirova, E. Karakoleva, and T. Stoyanchov, "ZnO thin film on side polished optical fiber for gas sensing applications," Appl. Surface Sci., vol. 254, no. 4, pp. 1087-1090, 2007.

[11] F. De-Jun, Z. Mao-Sen, G. X. Liu, L. Xi-Lu, and J. Dong-Fang, "D-shaped plastic optical fiber sensor for testing refractive index," IEEE Sensors J., vol. 14, no. 5, pp. 1673-1676, May 2014.

[12] X. Wang, G. Farrell, E. Lewis, K. Tian, L. Yuan, and P. Wang, "A humidity sensor based on a singlemode-side polished multimode-singlemode (SSPMS) optical fibre structure coated with gelatin," J. Lightw. Technol. vol. 35, no. 18, pp. 4087-4094, Sep. 2017.

[13] Q. Wu, Y. Semenova, P. Wang, and G. Farrell, "High sensitivity SMS fiber structure based refractometer-analysis and experiment," Opt. Express, vol. 19, no. 9, pp. 7937-7944, 2011.

[14] W. S. Mohammed, P. W. E. Smith, and X. J. Gu, "All-fiber multimode interference bandpass filter," Opt. Lett., vol. 31, no. 17, pp. 2547-2549, Sep. 2006

[15] A. M. Hatta, G. Farrell, P. Wang, G. Rajan, and Y. Semenova, "Misalignment limits for a singlemode-multimode-singlemode fiber-based edge filter," J. Lightw. Technol., vol. 27, no. 13, pp. 2482-2488, Jul. 2009.
[16] Q. Wu, Y. Semenova, P. Wang, and G. Farrell, "High sensitivity SMS fiber structure based refractometer-analysis and experiment," Opt. Express, vol. 19, no. 9, pp. 7937-7944, 2011.

[17] A. M. Hatta, G. Rajan, Y. Semenova, and G. Farrell, "SMS fibre structure for temperature measurement using a simple intensity-based interrogation system," Electron. Lett., vol. 45, no. 21, pp. 1069-1071, 2009.

[18] J. L. An, Y. X. Jin, M. M. Sun, and X. Y. Dong, "Relative humidity sensor based on SMS fiber structure with two waist-enlarged tapers," IEEE Sensors J., vol. 14, no. 8, pp. 2683-2686, Aug. 2014.

[19] K. Tian et al., "A curvature sensor based on twisted single-modemultimode-single-mode hybrid optical fibre structure," J. Lightw. Technol., vol. 35, no. 9, pp. 1725-1731, May 2017.

[20] P. Wang, G. Brambilla, M. Ding, Y. Semenova, Q. Wu, and G. Farrell, "High-sensitivity, evanescent field refractometric sensor based on a tapered, multimode fiber interference," Opt. Lett., vol. 36, no. 12, pp. 2233-2235, 2011.

[21] W. Han, Z. Tong, and Y. Cao, "Simultaneous measurement of temperature and liquid level base on core-offset singlemode-multimode-singlemode interferometer," Opt. Commun., vol. 321, pp. 134-137, 2014.

[22] J. Tang et al., "Fabrication of side-polished single mode-multimodesingle mode fiber and its characteristics of refractive index sensing," IEEE J. Sel. Topics Quantum Electron., vol. 23, no. 2, Mar./Apr. 2017, Art. no. 5600708.

[23] A. Gaston, F. Perez, and J. Sevilla, "Optical fiber relative-humidity sensor with polyvinyl alcohol film," Appl. Opt., vol. 43, no. 21, pp. 4127-4132, Jul. 2004.

[24] J. Mathew, Y. Semenova, and G. Farrell, "Effect of coating thickness on the sensitivity of a humidity sensor based on an Agarose coated photonic crystal fiber interferometer," Opt. Express, vol. 21, no. 5, pp. 6313-6320, Mar. 2013.

[25] K. M. Tan, C. M. Tay, S. C. Tjin, C. C. Chan, and H. Rahardjo, "High relative humidity measurements using gelatin coated long-period grating sensors," Sensors Actuators B, Chem., vol. 110, no. 2, pp. 335-341, Oct. 2005.

[26] P.-G. Su, Y.-L. Sun, and C.-C. Lin, "Humidity sensor based on PMMA simultaneously doped with two different salts," Sensors Actuators B, Chem., vol. 113, no. 2, pp. 883-886, 2006.

[27] J. Mathew, Y. Semenova, G. Rajan, P. F. Wang, and G. Farrell, "Improving the sensitivity of a humidity sensor based on fiber bend coated with a hygroscopic coating," Opt. Laser Technol., vol. 43, no. 7, pp. 1301-1305, Oct. 2011.

[28] I. D. Villar, A. B. Socorro, J. M. Corres, F. J. Arregui, and I. R. Matias, "Refractometric sensors based on multimode interference in a thin-film coated single-mode-multimode-single-mode structure with reflection configuration," Appl. Opt., vol. 53, no. 18, pp. 3913-3919, 2014.

[29] Y. Cardona-Maya, I. D. Villar, A. B. Socorro, J. M. Corres, I. R. Matias, and J. F. Botero-Cadavid, "Wavelength and phase detection based SMS fiber sensors optimized with etching and nanodeposition," J. Lightw. Technol., vol. 35 , no. 17 , pp. 3743-3749, Sep. 2017.

[30] G. Farrell, G. Brambilla, M. Ding, P. Wang, Q. Wu, and Y. Semenova, "Investigation of single-mode-multimode-single-mode and singlemode-tapered-multimode-single-mode fiber structures and their application for refractive index sensing," J. Opt. Soc. Amer. B, vol. 28, no. 5, pp. 1180-1186, 2011.

[31] K. Tiefenthaler and W. Lukosz, "Grating couplers as integrated optical humidity and gas sensors 放, Thin Solid Films, vol. 126, no. 3/4, pp. 205-211, 1985 .

Authors' biographies not available at the time of publication. 